\title{
Representation of involuted semigroups by binary relations
}

by

\author{
B. M. Schein (Saratov)
}

Abstract. We give necessary and sufficient conditions for an (ordered) involuted semigroup to be isomorphic with an (ordered) involuted semigroup of binary relations in which the involution is the conversion of relations (and the order is the set-theoretical inclusion). The same problem is solved for representations of (ordered) semigrouds (or semiheaps) by binary relations. The conditions of representability are universal Horn formulas.

An involuted semigroup is an algebra $A=\left(A ; \cdot,^{-1}\right)$ with a binary multiplication - and unary involution ${ }^{-1}$ subject to the following three identities:

$$
\begin{gathered}
(x y) z=x(y z) \\
\left(x^{-1}\right)^{-1}=x \\
(x y)^{-1}=y^{-1} x^{-1}
\end{gathered}
$$

A subalgebra of an involuted semigroup $A$ is called an involuted subsemigroup of $\boldsymbol{A}$.

Historically, the first example of involuted semigroups was that of groups. Every group is isomorphic to an involuted semigroup of permutations of a set (the Cayley theorem). Here a permutation of a set $X$ is a bijective self-mapping of $X$, i.e. a one-to-one and full binary relation on $X$. The multiplication and involution of permutations have their usual meaning of composition and conversion.

If in the definition of permutations we do not demand that they should be full, i.e. everywhere defined, we obtain involuted semigroups of partial one-to-one transformations of a set. Such involuted semigroups are inverse semigroups and every inverse semigroup is isomorphic to an involuted semigxoup of one-to-one partial transformations of a set (the Vagner-Preston theorem).

If in the definition of permutations we do not demand that they should be one-to-one, we obtain full multi-valued transformations, i.e. full 
binary relations of a set (in effect, since involuted semigroups are closed under involution, an involuted semigroup of binary relations contains converses of all the relations belonging to it; if at least one of the relations is not one-to-one, the involuted semigroup contains a multi-valued transformation). Those involuted semigroups which are isomorphic to involuted semigroups of full binary relations form an extensive class of algebras containing, in particular, all groups and all inverse semigroups. An axiomatization of this class was found in [12]. In particular, an involuted semigroup is an inverse semigroup if and only if it is isomorphic to an involuted semigroup of full binary relations and satisfies the identity [12] and [13]

$$
x x^{-1} x=x .
$$

Now, if in the definition of permutations we do not demand either oneto-oneness or fullness, we obtain arbitrary binary relations. An involuted semigroup of binary relations is an involuted semigroup of the form $(F ; \circ,-1)$ where $F$ is a nonempty set of binary relations on a set, $\circ$ is the relative multiplication and ${ }^{-1}$ is the usual conversion of binary relations.

Thus involuted semigroups of binary relations are, in a sense, an ultimate generalization of permutation groups. The main problem solved in this paper is an explicit abstract characterization of involuted semigroups of binary relations. In other words, we give a system of axioms necessary and sufficient in order for an abstract involuted semigroup to be isomorphic to an involuted semigroup of binary relations. This problem is similar to the problem of characterizing relation algebras in the sense of A. Tarski [17] or relation algebras in the sense of B. Jónsson [7]. An involuted semigroup isomorphic to an involuted semigroup of binary relations is called representable. It is known that the class of representable involuted semigroups forms a quasivariety. (i.e. it can be characterized by a system of elementary axioms each of which is a conditional identity) and not every involuted semigroup is representable [14]. It is shown in the present paper that the class of all representable involuted semigroups does not form a variety of algebras, i.e. cannot be characterized by a set of identities.

As a first step we solve the problem of abstract characterization of representable ordered involuted semigroups. An ordered involuted semigroup is an algebraic system of the form $(A ; \cdot,-1, \leqslant)$ where $\left(A ; \cdot,^{-1}\right)$ is an involuted semigroup and $(A ; \leqslant)$ is a (partially) ordered set, where the order relation $\leqslant$ is stable under both operations of the involuted semigroup, i.e. satisfies the axioms:

$$
\begin{gathered}
x_{1} \leqslant x_{2} \& y_{1} \leqslant y_{2} \Rightarrow x_{1} y_{1} \leqslant x_{2} y_{2}, \\
x \leqslant y \Rightarrow x^{-1} \leqslant y^{-1} .
\end{gathered}
$$

Since every set of binary relations is ordered by the set-theoretical inclusion of relations, we may consider ordered involuted semigroups of binary relations $\left(F ; \circ,{ }^{-1}, C\right)$; clearly, they satisfy conditions (5) and (6). An ordered involuted semigroup is called representable if it is isomorphic to an ordered involuted semigroup of binary relations.

The method used to solve the problem of characterization of representable involuted semigroups permits us to solve a similar problem of characterizing representable semigrouds. This problem is considered by many authors as the central problem of the theory of semigrouds.

A semigroud (in other terminology, a semiheap) is an algebra $(A$; [ ]) with a ternary operation $\left(a_{1}, a_{2}, a_{3}\right) \mid \rightarrow\left[a_{1} a_{2} a_{3}\right]$ satisfying the following identities of skew associativity

$$
\left.\mid\left[x_{1} x_{2} x_{3}\right] x_{4} x_{5}\right]=\left[x_{1}\left[x_{4} x_{3} x_{2}\right] x_{5}\right]=\left[x_{1} x_{2}\left[x_{3} x_{4} x_{5}\right]\right] .
$$

Semigrouds are closely connected with involuted semigroups. In fact, if $(A ; \cdot,-1)$ is an involuted semigroup and $\left[a_{1} a_{2} a_{3}\right]=a_{1} a_{2}^{-1} a_{3}$, then $(A ;[])$ is a semigroud and every semigroud can be isomorphically embedded into a semigroud associated with an involuted semigroup [2]. An ordered semigroud is an algebraic system of the form $(A ;[], \leqslant)$ where $(A ;[])$ is a semigroud, $(A ; \leqslant)$ is a (partially) ordered set and the order relation $\leqslant$ is stable under the ternary multiplication [ ]:

$$
x_{1} \leqslant x_{2} \& y_{1} \leqslant y_{2} \& z_{1} \leqslant z_{2} \Rightarrow\left[x_{1} y_{1} z_{1}\right] \leqslant\left[x_{2} y_{2} z_{2}\right] \text {. }
$$

Let $\varrho, \sigma, \tau \subset X \times Y$ be binary relations between the elements of two sets $X$ and $Y$. Define $[\varrho \sigma \tau]=\tau \circ \sigma^{-1} \circ \varrho$ (the factors in the product of

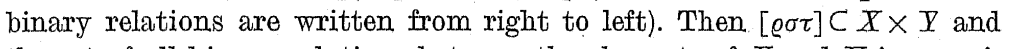
the set of all binary relations between the elements of $X$ and $Y$ is a semigroud under the ternary multiplication [ ]. Subsemigrouds of such semigrouds (for arbitrary $X$ and $X$ ) are called semigrouds of binary relations. If $(F ;[])$ is a semigroud of binary relations, then $(F ;[], C)$ is an ordered semigroud of binary relations (here $C$ is the set-theoretical inclusion relation on $F^{\prime}$ ). An (ordered) semigroud is called representable if it is isomorphic to an (ordered) semigroud of binary relations. It is known [14] that the class of all representable semigrouds forms a quasivariety and not every semigroud is representable. The problems of characterizing representable involuted, semigroups and semigrouds are long-standing ones (see [2], where the concept of a semigroud was introduced for the first time, and Problem T21 in [19]).

The problems of abstract characterizations of representable (ordered) involuted 'semigroups and semigrouds are typical problems on relation algebras in the sense of [10] and [11]. Representations of semigrouds by binary relations have been considered in [4], where a system of axioms 
for representable semigrouds has been found. However, these axioms are sentences in the third order predicate calculus. By the fundamental theorem on relation algebras, [10] and [11], the class of representable semigrouds is elementarily axiomatizable. We find a system of elementary axioms for this class.

A system of elementary axioms for representable involuted semigroups has been found by R. McKenzie [8] (never published). However, these axioms have a very complicated form and are not conditional identities. Our axioms have a much simpler form. Elementary axioms for representable ordered involuted semigroups have been found by D. A. Bredihin [1]. These axioms are similar to ours; however, our axioms are visualized much more easily.

Abstract characterizations for (ordered) involuted semigroups of special binary relations have been found in [5], [12], [13] and [16].

Abstract characterizations of (ordered) involuted semigroups of all binary relations on a set and of semigrouds of all binary relations between the elements of two sets have been found in [3], [6], [9] and [14] (in the case of semigroups) and in [14] (in the case of semigrouds). A related problem has been solved in [15].

The following concept of net is of central importance in this paper. A net of order $n$ is a finite oriented graph $M_{n}$ having $n+1$ vertices (which we identify with the natural numbers $\{0,1, \ldots, n\}$ ), having no loops, having an arrow $(0,1)$ (here $(i, j)$ denotes an arrow, i.e. an oriented edge, leadirg from $i$ to $j$ ) but not an arrow (1,0), and such that for every vertex $i>1$ there exists a uniquely determined arrow $\left(p_{i}, i\right)$ leading from $\{0,1, \ldots, i-1\}$ to $i$ and a uniquely determined arrow $\left(i ; q_{i}\right)$ leading from $i$ to $\{0,1, \ldots, i-1\}$ (thus, $p_{i}<i$ and $q_{i}<i$ ).

Since nets are extensively used in what follows, we will consider this concept in more detail.

First of all, it is clear that if $M_{n}$ is a net and $0<k \leqslant n$, then the set $\{0,1, \ldots, k\}$ of vertices and the set of all arrows of $M M_{n}$ between these vertices form a net $M_{k}$ of order $k$. A net $M$ is called a subnet of a net $M_{n}$ if $M=M_{k}$ for some $k, 0<k \leqslant n$. Thus $M_{n}$ has precisely $n$ subnets, namely, $M_{1}, M_{2}, \ldots, M_{n}$.

Any net $M_{n}$ of order $n$ has exactly $2 n-1$ arrows. In effect, the snbnet $M_{1}$ of $M_{n}$ has one arrow, by definition. Suppose the subnet $M_{i-1}$ has $2 i-3$ arrows. The subnet $M_{i}$ is formed from $M_{i-1}$ by adding a new vertex $i$ and two new arrows $\left(p_{i}, i\right)$ and $\left(i, q_{i}\right)$. Thus $M r_{i}$ contains $2 i-3+2=2 i-1$ arrows. By induction, $M_{n}$ contains $2 n-1$ arrows.

We shall number consecutively the arrows of any net of order $n$. The arrow $(0 ; 1)$ has number 0 . The arrows $\left(p_{i}, i\right)$ and $\left(i, q_{i}\right)$ have numbers $2 i-3$ and $2 i-2$ respectively. Thus every arrow is numbered by a single number from $\mathbf{0}$ to $\mathbf{2 n - 2}$. We identify the arrows with their numbers; to distinguish them from vertices, arrows are written as bold face numbers. E.g. $\left(p_{i}, i\right)=2 i-3$.

All nets can be constructed by a very simple procedure. The only existing net $M_{1}$ of order 1 is just two vertices 0 and 1 and an arrow $\mathbf{0}=(0,1)$. Any net of order $n$ is obtained from a net $M_{n-1}$ of order $n-1$ by adding a new vertex $n$ and two new arrows $\mathbf{2 n - 3}=\left(p_{n}, n\right)$ and $\mathbf{2 n - 2}=\left(n, q_{n}\right)$ where $p_{n}$ and $q_{n}$ are some (possibly coinciding) fixed vertices of $M_{n-1}$. This may be visualized in Fig. 1 .

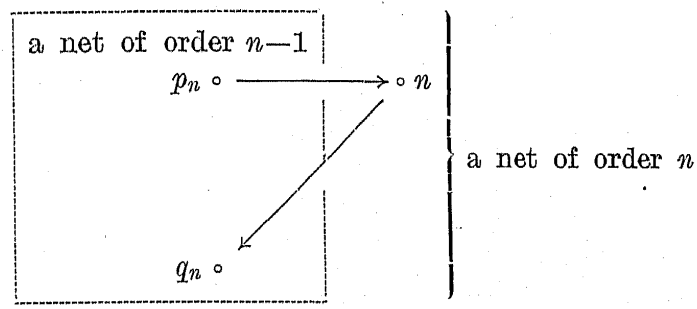

Fig. 1

Thus, a net of order $n$ can be constructed from a net $M_{n-1}$ of order $n-1$ in $n^{2}$ different ways. It follows that there exist $1 \cdot 2^{2} \cdot \ldots \cdot n^{2}=(n !)^{2}$ different nets of order $n$.

If $(i, j)$ is an arrow, then $(i, j)^{-1}$ is called the reversed arrow. The reversed net $M_{n}^{-1}$ is obtained from $M_{n}$ if all the arrows of $M_{n}$ are reversed. $(i, j)^{-1}$ leads from $j$ to $i$.

An edge of $M_{n}$ is either an arrow of $M_{n}$ or an arrow of $M_{n}^{-1}$ (i.e. an edge is either an arrow or a reversed arrow of $M_{n}$ ). A chain in $M_{n}$ is a nonempty finite sequence of edges such that the end of each edge coincides with the beginning of the next edge.

Let $A=(A ; \cdot,-1, \leqslant)$ be an ordered involuted semigroup and let $M_{n}$ be a net of order $n$. A metric on $M_{n}$ (more precisely, an $A$-metric on $M_{n}$ ) is any mapping $d:\{0, \mathbf{1}, \ldots, \mathbf{2} n-\mathbf{2}\} \rightarrow A$ of the set of arrows of $M_{n}$ into $A$.

Define $d\left(i^{-1}\right)=d(i)^{-1}$ for every arrow $i$ of $M_{n}$. If $\mu=\left(e_{1}, e_{2}, \ldots, e_{m}\right)$ is a chain in $M_{u}$ consisting of edges $e_{i}$, write $d(\mu)=d\left(e_{1}\right) d\left(e_{2}\right) \ldots d\left(e_{m}\right)$, where the right-hand side product is taken inside $A$. Then $d(\mu)$ is called the length of $\mu$.

An $A$-metric on, $M_{n}$ is called proper if for any $k, 0<k<n$, there exists a chain $\mu_{k}$ in $M_{k}$ leading from $p_{k+1}$ to $q_{k+1}$ whose length is shorter than that of the chain $(2 k-1,2 k)$, i.e. $d\left(\mu_{k}\right) \leqslant d(2 k-1) d(2 k)$.

Proper metrics on $I M_{n}$ can be constructed inductively in a simple way. We can define $d$ consecutively on subnets $M_{1}, M_{2}, \ldots, M_{n}$. A proper metric on $M_{1}$ is merely a mapping of $\{0\}$ into $A$, i.e. to define it we should 
pick out an element $a_{0} \in A$ and suppose $d(0)=a_{0}$. Suppose $d$ is defined on $M_{k}$. Choose a chain $\mu_{k}$ in $M_{k}$ leading from $p_{k+1}$ to $q_{k+1}$ and pick out two elements $a_{2 k-1}, a_{2 k} \in A$ such that $d\left(\mu_{k}\right) \leqslant a_{2 k-1} a_{2 k_{k}}$. Then define $d(\mathbf{2 k - 1})=a_{2 k-1}, d(\mathbf{2 k})=a_{2 k}$. The resulting $d$ is a proper metric on $M_{k+1}$. Thus we can construct a proper metric on $M_{n}$ and every proper metric on $M_{n}$ may be constructed in this fashion. We have supposed that for every $a \in A$ there exist $b, c \in A$ such that $a \leqslant b c$. However, as we shall see later, this is always the case in those ordered involuted semigroups in which we are interested.

A proper $\boldsymbol{A}$-metric $d$ on $M_{n}$ is called regular if for every chain $\mu$ in $M_{n}$ leading from 0 to $1 d(0) \leqslant d(\mu)$.

THEOREM 1. An ordered involuted semigroup $A$ is representable if and only if every proper $\boldsymbol{A}$-metric on every net is regular.

Proof. Necessity. Suppose $d$ is a proper $\boldsymbol{F}$-metric on a net $M_{n}$ of order $n$ for an ordered involuted semigroup $F$ of binary relations on a set $X$. Let $\left(x_{0}, x_{1}\right) \in d(0)$ for $x_{0}, x_{1} \in X$.

We are going to define a sequence $x_{0}, x_{1}, \ldots, x_{n}$ of elements of $X$ such that if $(i, j)$ is an arrow of $M_{n}$, then $\left(x_{i}, x_{j}\right) \in d((i, j))$.

The first two terms of such a sequence have already been given. Suppose $x_{0}, \ldots, x_{k}$ have already been defined $(0<k \leqslant n)$. By supposition, there exists a chain $\mu_{k}$ leading from $p_{k+1}$ to $q_{k+1}$ and such that $d\left(\mu_{k}\right)$ $C d(2 k-1) d(2 k)$. By the supposition of induction, $\left(x_{i}, x_{j}\right) \in d((i, j))$ for $0<i, j \leqslant k$. Therefore, $\left(x_{j}, x_{i}\right) \in d((i, j))^{-1}=d\left((i, j)^{-1}\right)$. By the definitions of a chain and the relative multiplication, $\left(x_{i}, x_{j}\right) \in d(v)$, where $v$ is any chain in $\boldsymbol{M}_{k}$ leading from $i$ to $j$. In particular, $\left(x_{p_{k+1}}, x_{q_{k+1}}\right) \in d\left(\mu_{k}\right)$ $C d(\mathbf{2 k - 1}) d(\mathbf{2 k})$. (Notice that here we do not use o to denote relative multiplication and the factors in the relative product of two binary relations are written from left to right and not from right to left as we do when $\circ$ is used). It follows that $\left(x_{p_{k+1}}, x_{k+1}\right) \in d(\mathbf{2 k - 1})$ and $\left(x_{k+1}, x_{q_{k+1}}\right)$ $\epsilon d(\mathbf{2 k})$ for some $x_{k+1} \in X$. Choose such $x_{k+1}$. Clearly, now $\left.\left(x_{i}, x_{j}\right) \in d(i, j)\right)$ for $0<i, j \leqslant k+1$. By induction, the whole sequence $x_{0}, \ldots, x_{n}$ can be constructed.

Now suppose $\mu$ is a chain in $M_{n}$ leading from 0 to 1 . As we have just seen, this implies $\left(x_{0}, x_{1}\right) \in d(\mu)$. Thus $\left(x_{0}, x_{1}\right) \in d(\mathbf{0})$ inplies $\left(x_{0}, x_{1}\right)$ $\epsilon d(\mu)$ for all $x_{0}, x_{1} \in X$, i.e. $d(0) \subset d(\mu)$. Therefore, the metric $d$ is regular.

Now if $\boldsymbol{A}$ is a representable ordered involuted semigroup, then every proper $\boldsymbol{A}$-metric is regular since this is true for all proper metrics with values in the ordered involuted semigroup of binary relations which is an isomorphic image of $A$.

Sufficiency. Let $K$ denote the class of all representable ordered involuted semigroups. Suppose $\boldsymbol{A}$ is an ordered involuted semigroup and every proper $A$-metric on every net is regular. Then this is true for all proper $\boldsymbol{B}$-metrics where $\boldsymbol{B}$ is any ordered involuted subsemigroup of $\boldsymbol{A}$ (since $\boldsymbol{B}$-metrics can be considered merely as a particular case of $\boldsymbol{A}$-metrics with values in $\boldsymbol{B})$. Suppose our theorem is true for denumerable ordered involuted semigroups. Then every finitely generated ordered involuted subsemigroup $\boldsymbol{B}$ of $\boldsymbol{A}$ satisfies the conditions of the theorem, i.e. $\boldsymbol{B} \in \pi$. It is known [10], [11] and [14] that the class $\pi$ is universal. Therefore [18], $K$ has the local property: if every finitely generated subsystem of a system belongs to $\pi$, the system itself belongs to $\pi$. So $A \in K$. It follows that we need to prove the theorem for the denumerable case only. Therefore, we suppose that $\boldsymbol{A}$ is a denumerable ordered involuted semigroup satisfying the condition of our theorem.

We are going to construct sequences $M_{1}<M_{2}<\ldots$ of nets and $d_{1} \subset d_{2} \subset \ldots$ of proper $A$-metrics (here $d_{i}$ is a metric on $M_{i}, M_{i-1}<M_{i}$. means that $M_{i-1}$ is a subnet of $M_{i}, d_{i-1} \subset d_{i}$ means $d_{i-1}$ is a restriction of $d_{i}$ ) satisfying the following condition $(\mathrm{P})$ :

(P) for every chain $\mu_{i k}$ in $M_{m}$ leading from $i$ to $k$ and for every $a_{1}, a_{2} \in A$ such that $d_{m}\left(\mu_{i k}\right) \leqslant a_{1} a_{2}$ there exist an $n$ and a vertex $j$ in $M_{n}$ such that $m \leqslant n$ and $d_{n}((i, j)) \leqslant a_{1}, d_{n}((j, k)) \leqslant a_{2}$.

Let $N$ be the set of all natural numbers, and let $(N \times N)^{*}$ denote the set of all finite sequences of pairs of natural numbers. Theh the set $(N \times N)^{*} \times A \times A$ is infinitely denumerable, so let $a$ be a fixed bijection of this set onto $N$.

We define $M_{n}, d_{n}$ inductively. Choose an element $a \in A$ and define $d(\mathbf{0})=a$. Then $d_{1}$ is a proper $A$-metric on $M_{1}$.

Suppose that we have defined the sequences $M_{1}<\ldots<M_{n-1}$ of nets and $d_{1} \subset \ldots \subset d_{n-1}$ of metrics. Let $R_{n-1}=\left\{\alpha\left(\mu, a_{1}, a_{2}\right): \mu\right.$ be a chain in $M_{n-1}$ leading, say, from $i$ to $k ; a_{1}, a_{2} \in A ; d_{n-1}(\mu) \leqslant a_{1} a_{2}$; there exists no vertex $j$ in $M M_{n-1}$ such that $d_{n-1}((i, j)) \leqslant a_{1}$ and $\left.d_{n-1}((j, k)) \leqslant a_{2}\right\}$. Notice that any chain in $M_{n-1}$ is a sequence of edges which are ordered pairs of natural numbers (vertices), and so every chain is an element of $(N \times N)^{*}$ and. the definition. of $R_{n-1}$ makes sense.

Two cases are possible.

Case 1. $R_{n-1}$ is empty. This means that the sequences $M_{1}<\ldots$ $\ldots<M_{n-1}$ and $d_{1} \subset \ldots \subset d_{n-1}$ satisfy condition $(\mathrm{P})$ and we need not prolong the sequences.

Case 2. $R_{n-1}$ is not emptiy. In this case define $\beta_{n-1}=\min R_{n-1}$. Then $\beta_{n-1}=\alpha\left(\mu_{t k}, a_{1}, a_{2}\right)$, where $\mu_{i k}$ is a chain in $M_{n-1}$ leading from $i$ to $k, a_{n-1}\left(\mu_{i k}\right) \leq a_{1} a_{2}$ and there is no vertex $j$ in $M_{n-1}$ such that $d_{n-1}((i, j))$ $\leqslant a_{1}$ and $d_{n-1}((j, k)) \leqslant a_{2}$. Define $M_{n}$ and $d_{n}$ as follows: add a new vertex $n$ and two new arrows $(i, n),(n, k)$ to $M_{n-1}$ and extend $d_{n-1}$ to $d_{n}$ supposing $d_{n}((i, n))=a_{1}, d_{n}((n, k))=a_{2}$. Clearly, $d_{n}$ is a proper metric on $M_{n}$. 3 - Fundamenta Mathematicae, T. Lxxxir 
Proceeding as above, we either obtain finite sequences satisfying property (P) or find that the sequences $M_{1}<\ldots$ and $d_{1} \subset \ldots$ are infinite. However, in the latter (infinite) case property $(\mathrm{P})$ is satisfied. In effect, it follows from the definition of $M_{n}$ that $\beta_{n-1}<\beta_{n}$; therefore, $n-1 \leqslant \beta_{n}$. Suppose $d_{m}\left(\mu_{i k}\right) \leqslant a_{1} a_{2}$ for a chain $\mu_{i k}$ in $M_{m}$ leading from $\ddot{i}$ to $k$ and for $a_{1}, a_{2} \in A$. Let $a\left(\mu_{i k}, a_{1}, a_{2}\right)=p$ and $n=p+2$. Then, by the minimality of $\beta_{n}$ and the evident inequality $\beta_{n}>p$, there exists a vertex $j$ in $M_{n}$ such that $a_{n}((i, j)) \leqslant a_{1}$ and $d_{n}((j, k)) \leqslant a_{2}$.

Let $X_{a}$ be the set of all vertices of the nets $M_{i}$ from the sequence constructed above (so, if the sequence is finite, $X_{a}$ is an initial sequence of $N$, otherwise, $X_{a}$ coincides with $\left.N\right)$. For every $b \in A$ define a binary relation $P_{a}(b)$ on $X_{a}$ as follows: $(i, j) \in P_{a}(b)$ if and only if there exists a net $M_{n}$ in our sequence and a chain $\mu_{i j}$ in $M_{n}$ leading from $i$ to $j$ such that $d_{n}\left(\mu_{i j}\right) \leqslant b$.

The following chain of equivalences shows that $P_{a}\left(b^{-1}\right)=P_{a}(b)^{-1}$ : $(i, j) \in P_{a}\left(b^{-1}\right) \Leftrightarrow d_{n}\left(\mu_{i j}\right) \leqslant b^{-1} \Leftrightarrow d_{n}\left(\mu_{i j}\right)^{-1} \leqslant b \Leftrightarrow d_{n}\left(\mu_{i j}^{-1}\right) \leqslant b \Leftrightarrow(j, i) \in P_{a}(b)$. Here we have used successively the definition of $P_{a}\left(b^{-1}\right),(6)$, the definition of the length of a chain and the fact that $\mu_{i j}^{-1}$ is a chain in $M_{n}$ leading from $j$ to $i$.

Now suppose $(i, j) \in P_{a}\left(a_{1}\right)$ and $(j, k) \in P_{a}\left(a_{2}\right)$. Then $d_{p}\left(\mu_{t j}\right) \leqslant a_{1}$ and $d_{q}\left(\mu_{j k}\right) \leqslant a_{2}$ for chains $\mu_{i j}$ in $M_{p}$ and $\mu_{j k}$ in $M_{q}$. Let $n=\max \{p, q\}$. Then $d_{n}\left(\mu_{i j}\right) \leqslant a_{1}$ and $d_{n}\left(\mu_{j k}\right) \leqslant a_{2}$. Using (5), we obtain: $d_{n}\left(\mu_{i j} \mu_{j k}\right)=d_{n}\left(\mu_{i j}\right) d_{n}\left(\mu_{j k}\right)$ $\leqslant a_{1} a_{2}$ and, since $\mu_{i j} \mu_{j k}$ is a chain in $M_{n}$ leading from $i$ to $k,(i, k) \in P_{a}\left(a_{1} a_{2}\right)$.

Conversely, suppose $(i, k) \in P_{a}\left(a_{1} a_{2}\right)$. Then $d_{m}\left(\mu_{i k}\right) \leqslant a_{1} a_{2}$ for some $m$ and a chain $\mu_{i k}$ in $M_{m}$ leading from $i$ to $k$. By property $(\mathrm{P})$, there exist an $n$ and a vertex $j$ in $M_{n}$ such that $d_{n}((i, j)) \leqslant a_{1}$ and $d_{n}((j, k)) \leqslant a_{2}$. Since $(i, j)$ and $(j, k)$ are chains in $M M_{n},(i, j) \in P_{a}\left(a_{1}\right)$ and $(j, 7) \in P_{a}\left(a_{2}\right)$. Therefore, $P_{a}\left(a_{1} a_{2}\right)=P_{a}\left(a_{2}\right) \circ P_{a}\left(a_{1}\right)$.

If $a_{1} \leqslant a_{2}$ and $(i, j) \in P_{a}\left(a_{1}\right)$, then $d_{n}\left(\mu_{i j}\right) \leqslant a_{1}$ for some $n$ and $\mu_{i j}$. It follows that $d_{n}\left(\mu_{i j}\right) \leqslant a_{2}$ and $(i, j) \in P_{a}\left(a_{2}\right)$. Thus $a_{1} \leqslant a_{2}$ implies $P_{a}\left(a_{1}\right)$ $C P_{a}\left(a_{2}\right)$.

Now let $X$ be the sum of the family $\left(X_{a}\right)_{a \epsilon_{A}}$ of sets. Without loss of generality we may suppose that the sets $X_{a}$ are disjoint (e.g. we may replace $X_{a}$ by $X_{a} \times\{a\}$ ). Define $P(b)=\Sigma\left(P_{a}(b)\right)_{a \epsilon_{A}}$ where $\Sigma$ denotes the disjoint union of $P_{a}(b)$ (if the sets $X_{a}$ are disjoint, $\Sigma$ is the ordinary settheoretical union). Clearly,

$$
\begin{gathered}
P\left(b^{-1}\right)=P(b)^{-1}, \\
P\left(a_{1} a_{2}\right)=P\left(a_{2}\right) \circ P\left(a_{1}\right)
\end{gathered}
$$

for all $b, a_{1}, a_{2} \in A$, since the same properties hold for $P_{a}$. In the same. way, $a_{1} \leqslant a_{2} \Rightarrow P\left(a_{1}\right) \subset P\left(a_{2}\right)$. Now suppose $P\left(a_{1}\right) \subset P\left(a_{2}\right)$ for some $a_{1}, a_{2} \in A$.
Then $P_{a}\left(a_{1}\right) \subset P_{a}\left(a_{2}\right)$ for all $a \in A$. In particular, $P_{a_{1}}\left(a_{1}\right) \subset P_{a_{1}}\left(a_{2}\right)$. Since $d_{1}((0,1))=a_{1}$, we conclude that $(0,1) \in P_{a_{1}}\left(a_{1}\right) ;$ hence $(0,1) \in P_{a_{1}}\left(a_{2}\right)$. Therefore, there exist an $n$ and a chain $\mu_{01}$ in $M_{n}$ leading from 0 to 1 and such that $d_{n}\left(\mu_{01}\right) \leqslant a_{2}$. By the condition of our theorem, the metric $d_{n}$ is regular. Therefore, $a_{1}=d_{n}(0) \leqslant d_{n}\left(\mu_{01}\right) \leqslant a_{2}$. Thus

$$
a_{1} \leqslant a_{2} \Leftrightarrow P\left(a_{1}\right) \subset P\left(a_{2}\right) \text {. }
$$

Formulas (9)-(1.1) show that $P$ is an isomorphism of $A$ onto an ordered involuted semigroup of binary relations. In effect, if $P\left(a_{1}\right)$ $=P\left(a_{2}\right)$, then $P\left(a_{1}\right) \subset P\left(a_{2}\right)$ and $P\left(a_{2}\right) \subset P\left(a_{1}\right)$. By (11), $a_{1} \leqslant a_{2}$ and $a_{2} \leqslant a_{1}$; hence $a_{1}=a_{2}$ and $P$ is one-to-one. The form of $(10)$ is due to the fact that we write factors in products from left to right in abstract semigroups (as in $\boldsymbol{A}$ ) and from right to left in semigroups of binary relations.

Thus the ordered involuted semigroup is representable, which ends the proof of Theorem 1.

Remark 1. Suppose that $A=(A ; \cdot,-1, \leqslant)$ is a quasiordered involuted semigroup, i.e. that $\leqslant$ is a quasiorder (that is, reflexive and transitive) binary relation on an involuted semigroup $(A ; \cdot,-1)$ and properties $(5)$ and (6) are satisfied. $\boldsymbol{A}$ is called representable in a wider sense if there exists a mapping $P$ of $\boldsymbol{A}$ onto an ordered involuted semigroup of binary relations satisfying (9)-(11). This being the case,

$$
P\left(a_{1}\right)=P\left(a_{2}\right) \Leftrightarrow\left(a_{1} \leqslant a_{2} \& a_{2} \leqslant a_{1}\right),
$$

i.e. $P$ is an isomorphism if and only if $\leqslant$ is an order relation. The same proof as that of Theorem 1 shows that a quasiordered involuted semigroup is representable in a wider sense if and only if it satisfies the condition of Theorem 1 (i.e. every proper metric on every net is regular). An obvious readjustment of the definition of proper metrics in the case of quasiorders is needed.

Remark 2. Let $\left(A ; \cdot,,^{-1}\right)$ be an involuted semigroup and let $\varrho$ be a binary relation on $A$. Define proper $\boldsymbol{A}$-metrics for $\boldsymbol{A}=(A ; \cdot,-1, \varrho)$ precisely as for ordered involuted semigroups, using $\varrho$ instead of $\leqslant$. Define $a_{1} \leqslant 0 a_{2}$ for $a_{1}, a_{2} \in A$. if and only if thero exist a net $M_{n}$ and a proper $A$-metric $d$ on $M_{n}$ such that $d(0)=a_{1}$ and $d(\mu)=a_{2}$ for some chain $\mu$ in $M_{n}$ leading from 0 to 1. Then $\leqslant_{0}$ is the smallest element in the set of all quasiorder relations $\pi$ on $A$ for which $\varrho \subset \pi$ (i.e. $a_{1} \varrho a_{2} \Rightarrow a_{1} \pi a_{2}$ for all $a_{1}, a_{2} \in A$ ) and $(A ; \cdot,-1, \pi)$ is a quasiordered involuted semigroup representable in a wider sense. The proof of this fact is quite analogous to the proofs of Theorems 1 and 2 .

Now we are going to characterize representable involuted semigroups. The sense of our necessary and sufficient condition of representability (Theorem 2) is quite simple: on an involuted semigroup $A$ 
we construct a quasiorder relation $\leqslant_{=}$(cf. Remark 2 ) which is the smallest quasiorder relation turning $\boldsymbol{A}$ into a quasiordered involuted semigroup representable in a wider sense; $\boldsymbol{A}$ is representable if and only if $\leqslant=$ is antisymmetric (i.e. $\leqslant=$ is an order. relation). In other words, $\boldsymbol{A}$ is representable if and only if for every two nets $M_{m}$ and $M_{n}$ and every two proper $\boldsymbol{A}$-metrics $d_{m}$ on $M_{m}$ and $d_{n}$ on $M_{n}$ (here in the definition of a proper metric the equality relation $=$ is used instead of $\leqslant$ ), every two chains $\mu$ in $M_{m}$ and $\nu$ in $M_{n}$, leading from 0 to 1 , the equalities $d_{m}(\mu)=d_{n}(0)$ and $d_{n}(v)=d_{m}(\mathbf{0})$ imply $d_{m}(\mathbf{0})=d_{n}(\mathbf{0})$. However, it turns out that we may assume $M_{m}=M_{n}$ in the above condition. Moreover, if every element of $A$ is decomposable into a product of two elements, the metrics $d_{m}$ and $d_{n}$ in the above condition may be chosen alike (in the sense that the chains figuring in the definition of $d_{m}$ are the same as the chains for $d_{n}$ ). To make all elements of $A$ decomposable we add a new element to $A$.

Let $A=\left(A ; \cdot,^{-1}\right)$ be an involuted semigroup. If the semigroup $(A ; \cdot)$ contains an identity 1 , then $1^{-1}=1$. In effect, $1^{-1}=1 \cdot 1^{-1}$ $=\left(1 \cdot 1^{-1}\right)^{-1}=\left(1^{-1}\right)^{-1}=1$. If $(A ; \cdot)$ does not contain an identity, adjoin a new element 1 to $A$ and define $1 \cdot x=x \cdot 1=x$ for all $x \in A \cup\{1\}, 1^{-1}=1$. As a result we obtain a new involuted semigroup with identity; this involuted semigroup will be denoted by $\boldsymbol{A}^{1}$. If $\boldsymbol{A}$ contains an identity, suppose $A^{1}=A$.

Suppose that $\boldsymbol{A}$ is an involuted semigroup, and $M_{n}$ is a net. An $A$-metric on $M_{n}$ is any mapping $d$ of the set $\{\mathbf{0}, \mathbf{1}, \ldots, \mathbf{2 n - 2}\}$ of all arrows of $M_{n}$ into $A$. Lengths of chains in any $A$-metric $d$ on $M_{n}$ are defined precisely as in the case of ordered involuted semigroups. An $\boldsymbol{A}$-metric $d$ on $M_{n}$ is called proper if for any $k, 0<k \leqslant n$, there exists a chain $\mu_{k}$ in $M_{k}$ leading from $p_{k+1}$ to $q_{k+1}$ whose length equals that of the chain $(\mathbf{2 k}-\mathbf{1}, \mathbf{2 k})$, i.e. $d\left(\mu_{k}\right)=d(\mathbf{2 k - 1}) d(\mathbf{2 k})$. Two proper $\boldsymbol{A}$-metrics $d$ and $d^{\prime}$ on $M_{n}$ are called similar if for any $k, 0<k<n$, there exists a chain $\mu_{k}$ in $M_{k}$ leading from $p_{k+1}$ to $q_{k+1}$ and such that $d\left(\mu_{k}\right)=d(\mathbf{2 k - 1}) d(\mathbf{2 k})$ $d^{\prime}\left(\mu_{k}\right)=d^{\prime}(\mathbf{2} k-\mathbf{1}) d^{\prime}(\mathbf{2} k)$. In other words, two proper $A$-metrics are. similar if the same chains can be used in ascertaining the properness of both metrics.

If $d$ is a metric on $\boldsymbol{A}$, then $d(\mathbf{0})$ is called the major value of $d$. Two metrics $d$ and $d^{\prime}$ on $M_{n}$ are called chained if there exists a chain $\mu$ in $M_{n}$ leading from 0 to 1 and such that $d(\mu)=d^{\prime}(0), d^{2}(\mu)=d(0)$.

THEOREM 2. An involuted semigroup $\boldsymbol{A}$ is representable if and only if every two chained similar proper $\boldsymbol{A}^{1}$-metrics on every net have the same major values.

Proof. Necessity. As in the proof of Theorem 1, it suffices to prove the condition of our theorem for an involuted semigroup $\boldsymbol{F}$ of binary relations. We shall prove a stronger condition: for every two nets $M_{m}$ and $M_{n}$ and chains $\mu$ in $M_{m}, v$ in $M_{n}$, both leading from 0 to 1 , and for every two proper $\boldsymbol{F}^{1}$-metrics, $d_{m}$ on $M_{m}$ and $d_{n}$ on $M_{n}$, the equalities $d_{m}(\mu)=d_{n}(0)$ and $d_{n}(\nu)=d_{m}(0)$ imply $d_{m}(0)=d_{n}(0)$.

Suppose that $M_{m}, M_{n}, \mu, \nu, d_{m}$ and $d_{n}$ are as above and the above two equalities hold. Since $\boldsymbol{F}^{1}$ is an involuted semigroup of binary relations $(*)$, it is ordered by the inclusion order relation $C$; let $\boldsymbol{F}^{\mathrm{C}}$ denote the corresponding ordered involuted semigroup. Now $d_{m}$ and $d_{n}$ may be considered as $\boldsymbol{F}^{\complement}$-metrics; obviously, both $d_{m}$ and $d_{n}$ are proper $\boldsymbol{F}^{\mathrm{C}}$ metrics. By Theorem $1, d_{m}(\mathbf{0}) \subset d_{m}(\mu)$ and $d_{n}(\mathbf{0}) \subset d_{n}(\nu)$; hence $d_{m}(0)$ $\subset d_{m}(\mu)=d_{n}(\mathbf{0})$ and analogously $d_{n}(\mathbf{0}) \subset d_{m}(\mathbf{0})$. It follows that $d_{m}(\mathbf{0})=d_{n}(\mathbf{0})$.

Sufficiency. One possible approach is to define a binary relation $\leqslant$ on the set $A$ of elements of an involuted semigroup $A: a_{1} \leqslant a_{2}$ if and only if there exist a net $M_{n}$, a proper $A^{1}$-metric $d$ on $M_{n}$ and a chain $\mu$ in $M_{n}$ leading from 0 to 1 and such that $a_{1}=d(0), a_{2}=d(\mu)$. Then we could prove that $\left(A ; \cdot,^{-1}, \leqslant\right)$ was an ordered involuted semigroup satisfying the condition of Theorem 1 . Thus $(A ; \cdot,-1, \leqslant)$ would be representable; hence. $A$ would be representable as well. However, such an approach leads to an entirely new proof as compared with that of Theorem 1. To save room we give another proof, using that of Theorem 1.

As in Theorem 1, we see that without loss of generality $A$ may be supposed to be a finite or countably infinite involuted semigroup satisfying the condition. of Theorem 2. Now copy the whole proof of Theorem 1 up to the formulas (9) and (10) with the following alterations: consider $\boldsymbol{A}^{1}$ instead of $A$ and $=$ instead of $\leqslant$. The proof is transferred to this case without alterations. Then $P$ is a homomorphism of $\boldsymbol{A}^{\mathbf{1}}$ onto an involuted semigroup of binary relations. It remains to prove that $P$ is an isomorphism, i.e. that $P$ is one-to-one.

Suppose $P\left(a_{1}\right)=P\left(a_{2}\right)$ for $a_{1}, a_{2} \in A$. Then $P_{a}\left(a_{1}\right)=P_{a}\left(a_{2}\right)$ for all $a \in A$. In particular, $P_{a_{1}}\left(a_{1}\right)=P_{a_{1}}\left(a_{2}\right)$. As in the proof of sufficiency of Theorem 1 , we obtain $(0,1) \in P_{a_{1}}\left(a_{2}\right)$, i.e. there exist an $n$ and a chain $\mu$ in $M_{n}$ leading from 0 to 1 such that $d_{n}(0)=a_{1}$ and $d_{n}(\mu)=a_{2}$ for a proper $A^{1}$-metric $d_{n}$ on $M_{n}$. Analogously, there exist an $m$, a chain $v$ in $M_{m}^{\prime}$ and a proper $\boldsymbol{A}^{1}$-metric $d_{m}^{\prime}$ on $M_{m}^{\prime}$ such that $d_{m}^{\prime}(0)=a_{2}$ and $d_{m}^{\prime}(v)=a_{1}$. Here condition (P) is satisfied for $M_{n}, d_{n}$ and for $M_{m}^{\prime}, d_{m}^{\prime}$.

We are going to construct a new net $M$ of order $m+n$ "glueing together" $M_{n}$ and. $M_{m}^{\prime}$ as in Fig. 2, where the arrows displayed in $M_{n}$ and $M_{m}^{\prime}$ are the 0 th arrows and a new arrow is added. The problem is to renumber the vertices and arrows of $M$.

The vertices of $M$ are $\{0,1, \ldots, m+n\}$, the arrows are $\{\mathbf{0}, \mathbf{1}, \ldots, \mathbf{2 m}+$ $+2 n-2\}$. Define injections $f$ and $g$ of the sets of vertices of $M_{n}$ and $M_{m}^{\prime}$ into the set of vertices of $M$. as follows: $f(0)=0, f(k)=k+1$ for $1 \leqslant k \leqslant n$;

(*) We suppose $F^{1}=F \cup\{\Delta\}$ where $\Delta$ is the identity relation in the case of $F^{1} \neq F$. 
$g(0)=2, g(1)=1, g(k)=k+n$ for $2 \leqslant k \leqslant m$. If $(i, j)$ is an arrow in $M_{n}$, then $(f(i), f(j))$ is an arrow in $M$, if $(i, j)$ is an arrow in $M_{m}^{\prime}$, then $(g(i), g(j))$ is an arrow in $M$. In other words, $f$ and $g$ are isomorphic embeddings of $M_{n}$ and $M_{m}^{\prime}$ into $M$. If $(i, j)$ is an arrow in $M_{n}$, then we will write $f((i, j))$ instead of $(f(i), f(j)) ; g((i, j))$ has an analogous meaning. $f((i, j))^{-1}$ $=(f(i), f(j))^{-1} ; g((i, j))^{-1}=(g(i), g(j))^{-1}$. If $\pi$ is a chain in $M_{n}$ (in $\left.M_{m}^{\prime}\right)$, then $f(\pi)(g(\pi))$ denotes the corresponding chain in $M$.

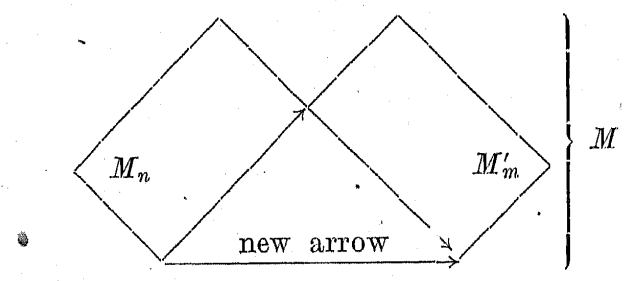

Fig. 2

Define $\boldsymbol{A}^{1}$-metrics $d$ and $d^{\prime}$ on $M$ as follows: $d(0)=a_{1}, d(f(i))=d_{n}(i)$ for every arrow $\boldsymbol{i}$ of $M_{n}, d|g(i)|=1$ for every arrow $\boldsymbol{i}$ of $\mathbb{I}_{m}^{\prime} ; d^{\prime}(\mathbf{0})=a_{2}$, $d^{\prime}(f(i))=1$ for every arrow $i$ of $M_{n}, d^{\prime}(g(i))=d_{m}^{\prime}(i)$ for every arrow $i$ of $M_{m}^{\prime}$. To check the fact that both $d$ and $d^{\prime}$ are proper we need only mention that $d(\mathbf{1}) d(\mathbf{2})=a_{1} \cdot 1=a_{1}=d(\mathbf{0}), d^{\prime}(\mathbf{1}) d^{\prime}(\mathbf{2})=1 \cdot a_{2}=a_{2}=d^{\prime}(\mathbf{0})$. Clearly, $d$ and $d^{\prime}$ are similar, since all the chains we used to show that $d_{n}$ and $d_{m}^{\prime}$ are proper are isomorphically mapped (by means of $f$ or $g$ ) into $M$. Since $d(f(\mu), g(v))=d(f(\mu)) d(g(v))=a_{2} \cdot 1=a_{2}=d^{\prime}(\mathbf{0})$ and $d^{\prime}((f(\mu), g(v))$ $=d^{\prime}\left(f(\mu)\left|d^{\prime}\right| g(\hat{\nu}) \mid=1 \cdot a_{1}=a_{1}=d(\mathbf{0})\right.$, the metrics $d$ and $d^{\prime}$ are chained. Here $(f(\mu), g(v))$ denotes the juxtaposing of the chains $f(\mu)$ and $g(\nu)$ in $M$. Clearly, it is a chain in $M$ leading from 0 to 1 .

By the condition of our theorem, $a_{1}=d(0)=d^{\prime}(0)=a_{2}$, i.e. $P$ is one-to-one. $P$ induces an isomorphism of $A$ onto an involuted semigroup of binary relations, i.e. $A$ is representable, which completes the proof of Theorem 2.

Suppose that $G$ is a set, $g \mid \rightarrow g^{*}$ is a bijection of $G$ onto a set $G^{*}, G$ and $G^{*}$ are disjoint, and $H=G \cup G^{*}$. The set $\boldsymbol{F I}\left(G^{*}\right)$ of all nonempty words over the alphabet $H$ is an involuted semigroup under the juxtaposition of words and the following involution: $\left(h_{1} h_{2} \ldots h_{n}\right)^{-1}=h_{n}^{-1} \ldots h_{2}^{-1} h_{1}^{-1}$ for all $h_{1}, h_{2}, \ldots, h_{n} \in H$. Here $g^{-1}=g^{*}$ and $\left(g^{*}\right)^{-1}=g$ for all $g \in G$.

$\boldsymbol{F I}(G)$ is a free involuted semigroup, and $G$ is a set of free generators of $\boldsymbol{F I}(G)$. We identify the identity of $\boldsymbol{F I}(G)^{1}$ with the empty word 1.

Proposition 3. Free involuted semigroups are representable.

Proof. Suppose that $\boldsymbol{F I}(G)$ is a free involuted semigroup, and $|\alpha|$ denotes the length of the word $\alpha \in \boldsymbol{F I}(G)^{1}$; in particular, $|1|=0$.
Let $M_{n}$ be a net, and let $d$ and $d^{\prime}$ be chained similar proper $\boldsymbol{F I}(G)^{1}$ metrics on $M_{n}, d(\mu)=d^{\prime}(0), d^{\prime}(\mu)=d(0)$ for a chain $\mu$ in $M_{n}$ leading.
from 0 to 1 .

We shall prove that $|d(\mathbf{0})| \leqslant|d(v)|$ for every chain $v$ leading from 0 to 1 , and $|d(\mathbf{0})|=|d(v)|$ implies $d(\mathbf{0})=d(v)$. Let $m$ be the minimal number such that $\nu$ is a chain in $M_{m}$. Then $m \leqslant n$. We give a proof by induction. If $m=1$, then $\nu$ is of the form $\left(\mathbf{0}, \mathbf{0}^{-1}, \mathbf{0}, \ldots, \mathbf{0}^{-1}, \mathbf{0}\right)$ and our statement is obviously true. Suppose it is true for all $m \leqslant k$. Let $v$ be a chain in $M_{k+1}$ but not in $M_{k}$. Then at least one of the edges $2 k-1,(2 k-1)^{-1}, 2 k,(2 k)^{-1}$ belongs to $\nu$. Suppose that $\mu_{k}$ is a chain in $M_{k}$ leading from $p_{k+1}$ to $q_{k+1}$ and such that $d\left(\mu_{k}\right)=d(\mathbf{2} \boldsymbol{k - 1}) \cdot d(\mathbf{2 k})$. Such a chain does exist since $d$ is proper. Replace every subchain $(2 k-\mathbf{1}, 2 k)$ occurring in $v$ by $\mu_{k}$ and every subchain $\left((2 k)^{-1},(2 k-1)^{-1}\right)=(2 k-1,2 k)^{-1}$ by $\mu_{k}^{-1}$. We obtain a chain $\bar{v}$ leading from 0 to 1 . Clearly, $d(v)=d(\bar{v})$. Omit all the occurrences of subchains $\left(\mathbf{2 k - 1},(\mathbf{2 k - 1})^{-1}\right),\left((\mathbf{2 k})^{-1}, \mathbf{2 k}\right)$ from $\bar{\nu}$. We obtain a chain $\overline{\bar{\nu}}$ leading from 0 to 1 and such that $|d(\overline{\bar{v}})| \leqslant|d(\bar{v})|$. Clearly, $|d(\overline{\bar{v}})|=|d(\bar{v})|$ if and only if $\bar{v}$ does not contain the above subchains, i.e. $\bar{v}=\overline{\bar{\nu}}$. Now, $\overline{\bar{\nu}}$ is a chain in $M_{k}$ and, by the supposition of induction, $|d(\mathbf{0})| \leqslant|d(\bar{\nu})|$. Therefore, $|d(\mathbf{0})| \leqslant|d(\nu)|$. If $|d(\mathbf{0})|=|d(\nu)|$, then $|d(\mathbf{0})|=|d(\overline{\bar{\nu}})|, v=\overline{\bar{\nu}}$ and, by the supposition of induction, $d(\mathbf{0})=d(\overline{\bar{v}})=d(\bar{v})=d(v)$.

Thus $|d(\mathbf{0})| \leqslant|d(\mu)|=\left|d^{\prime}(\mathbf{0})\right| \leqslant\left|d^{\prime}(\mu)\right|=|d(\mathbf{0})| ;$ hence, $|d(\mathbf{0})|=|d(\mu)|$ and, by the above statement, $d(\mathbf{0})=d(\mu)=d^{\prime}(\mathbf{0})$. Therefore, the condition of Theorem 2 is fulfilled. It follows that $\boldsymbol{F l}(G)$ is representable.

COROLLARY 4. The class of all representable involuted semigroups does not form a variety of algebras, since it is not closed under homomorphisms.

Proof. Every involuted semigroup is a homomorphic image of a free one; there exist involuted semigroups which are not representable [14]; free involuted semigroups are representable by Proposition 3.

Remark 3. It was proved in [14] and in [10] and [11] that the class of all representable involuted semigroups forms a quasivariety of algebras (i.e. a class characterized by a system of quasiidentities, a quasiidentity being a universal formula of the form $A_{1} \& A_{2} \& \ldots \& A_{n} \Rightarrow A$, where $A_{1}, A_{2}, \ldots, A_{n}, A$ are equations and no quantifiers occur). It follows from Corollary 4 that the class of all representable involuted semigroups cannot be characterized by a system of identities. Moreover, it follows from Proposition 3 that any identity that holds for all representable involuted semigroups holds for all involuted semigroups as well.

The conditions of representability given in Theorems 1 and 2 do not have the form of conditional identities. However, they can easily be transformed into conditional identities (i.e. quasiidentities), which we do in what follows.

Let $M_{n}$ be a net. Consider the first order predicate calculus fit for 
(ordered) involuted semigroups. The alphabet of the calculus comprises a countably infinite list of individual variables $x_{0}, x_{1}, \ldots$, two symbols for the operations of involuted semigroups (and a symbol for the order relation), logical symbols (propositional connectives, quantifiers), equality, technical symbols (brackets). With every arrow $i$ of $M_{n}$ associate the individual variable $x_{i}$. With every chain $\mu=\left(\boldsymbol{e}_{1}, \boldsymbol{e}_{2}, \ldots, \boldsymbol{e}_{m}\right)$ in $M_{n}$ as sociate the term (polynomial) $[\mu]$ as follows: $[\boldsymbol{i}]=x_{i},\left[\boldsymbol{i}^{-1}\right]=x_{i}^{-1},[\mu]$ $=\left[\boldsymbol{e}_{1}\right]\left[\boldsymbol{e}_{2}\right] \ldots\left[\boldsymbol{e}_{m}\right]$. For example, $\left[\left(\mathbf{0}, \mathbf{4}^{-1}, \mathbf{6}^{-1}, \mathbf{6}, \mathbf{3}^{-1}, \mathbf{1}\right)\right]=x_{0} x_{4}^{-1} x_{0}^{-1} x_{0} x_{3}^{-1} x_{1}$.

A chain system in $M_{n}$ is a sequence $\left(\mu_{1}, \mu_{2}, \ldots, \mu_{n-1}, \mu\right)$ where $\mu_{i}$ is a chain in $M_{i}$ leading from $p_{i+1}$ to $q_{i+1}, \mu$ is a chain in $I M_{n}$ leading from 0 to 1.

With every chain system $S$ in $M_{n}$ associate the following formula $\langle S\rangle$ : if $S=\left(\mu_{1}, \mu_{2}, \ldots, \mu_{n-1}, \mu\right)$, then $\langle S\rangle$ is

$$
\begin{gathered}
{\left[\mu_{1}\right] \leqslant x_{1} x_{2} \&\left[\mu_{2}\right] \leqslant x_{3} x_{4} \& \ldots \&\left[\mu_{i}\right] \leqslant x_{2 i-1} x_{2 i} \& \ldots} \\
\ldots \&\left[\mu_{n-1}\right] \leqslant x_{2 n-3} x_{2 n-2} \Rightarrow x_{0} \leqslant[\mu] .
\end{gathered}
$$

THEOREM 5. An ordered involuted semigroup is representable if and only if it satisfies conditions $\langle S\rangle$ for every chain system $S$ in every net $M_{n}$.

Proof. Suppose that $S$ is a chain system in a net $M_{n}$. Substitute an element $d(\boldsymbol{i})$ of an ordered involuted semigroup $\boldsymbol{A}$ for each occurrence of $x_{i}$ in $S$. Then $d$.can be considered as a metric on. $M_{n}$. If the antecedent of the implication $\langle S\rangle$ is valid, this means that $d$ is a proper $A$-metric (for the chains $\mu_{1}, \ldots, \mu_{n-1}$ occurring in $S$ ). The regularity of $d$ means that $d(\mathbf{0}) \leqslant d(\mu)$ for every chain $\mu$ in $M_{n}$ leading from 0 to 1 . In other words, $x_{0} \leqslant[\mu]$ for our assignment of values in $A$ for individual variables $x_{i}$. Thus $A$ satisfies the condition of Theorem 5 if and only if every proper $A$-metric on every net $M_{n}$ is regular. Now Theorem 5 follows from Theorem 1, which completes the proof.

Theorem 5 characterizes the class of all representable ordered involuted semigroups in terms of elementary axioms. Clearly, the system of axioms given in Theorem 5 is infinite. Moreover, for every net $M_{n}$ we can construct an infinite system of axioms, since there exists an infinite set of chain systems in $M_{n}$. The axioms obtained in this way are not independent. Roughly speaking, shorter axioms follow from longer ones. In what follows we reduce the number of axioms corresponding to a given net $M_{n}$.

A chain $\nu$ in $M_{n}$ is called straightened if it does not contain subchains of the form $(2 i-1,2 i)$ or $(2 i-1,2 i)^{-1}$.

RESTRICTION 1. In conditions $\langle S\rangle$ associated with chain systems we may consider straightened chains only.

Motivation. Suppose $\mu_{k}$ is a chain in a chain system $S$ and $\mu_{k}$ contains a subchain $(\mathbf{2} \boldsymbol{i}-\mathbf{1}, \mathbf{2} \boldsymbol{i})$. Clearly, $i<k$. The chain $\mu_{i}$ leads from $p_{i+1}$ to $q_{i+1}$ as well as the chain $(2 i-1,2 i)$. Replace $(2 i-\mathbf{1}, 2 i)$ by $\mu_{i}$ in $\mu_{k}$. We obtain a new chain $\bar{\mu}_{k}$. Now if the antecedent of $\langle S\rangle$ holds for some $x_{i} \in A$, then $\left[\mu_{i}\right] \leqslant x_{2 i-1} x_{2 i}$ and $\left[\mu_{k}\right] \leqslant x_{2 k-1} x_{2 k}$. By $(5),\left[\bar{\mu}_{k}\right] \leqslant x_{2 k-1} x_{2 k}$, since $\left[\bar{\mu}_{k}\right] \leqslant\left[\mu_{k}\right]$. Thus $\mu_{k}$ may be replaced by $\bar{\mu}_{k}$ in the antecedent of $\langle S\rangle$. Let $i$ be the smallest number such that the chain $\mu_{i}$ is not straightened. Clearly, $\mu_{1}$ is always straightened, and so $2 \leqslant i$. As we have just seen, we may replace $\mu_{l}$ (by replacing all occurrences of subchains of the form $(2 j-1,2 j)$ and $(2 j-1,2 j)^{-1}$ by $\mu_{\jmath}$ and $\left.\mu_{j}^{-1}\right)$ by another chain $\overline{\bar{\mu}}_{i}$ which is straightened. We obtain a new chain system $\bar{S}$ and condition $\langle\bar{S}\rangle$ implies $\langle S\rangle$. It is now easily seen that we can replace all non-straightened chains in the antecedent of $\langle S\rangle$ by straightened ones, and the resulting condition implies $\langle S\rangle$.

It remains to consider the final chain $\mu$ of $S$. If $\mu$ contains a subchain $(2 i-1,2 i)$ and we replace it by $\mu_{i}$, we obtain a new chain $\bar{\mu}$. If $x_{0} \leqslant[\bar{\mu}]$, then $[\bar{\mu}] \leqslant[\mu]$ and $x_{0} \leqslant[\mu]$. Therefore, if $\mu$ is replaced by $\bar{\mu}$ in $\langle S\rangle$, we obtain a stronger condition. It follows that we may consider the chain systems with straightened chains only.

Consider the net $M_{1}$. Every chain in $M_{1}$ is of the form $\left(0,0^{-1}, \mathbf{0}, \ldots\right.$ $\left.\ldots, 0^{-1}, 0\right)$ and every chain system in $M_{1}$ is of the form $(\mu)$. Therefore, we obtain the following axioms corresponding to $M_{1}: x_{0} \leqslant x_{0} x_{0}^{-1} x_{0} \ldots x_{0}^{-1} x_{0}$. In particular,

$$
x \leqslant x x^{-1} x
$$

(here $x_{0}$ is replaced by $x$ for convenience). Clearly, (12) implies every other axiom corresponding to $M_{1}$. By Theorem $5_{2}$, every representable ordered involuted semigroup satisfies (12).

Let $v$ be a chain in $M_{n}$. Then $\left(v, v^{-1}, v\right)$ is a chain which is called a zigzag. If a chain $\mu$ does not contain subchains which are zigzags, $\mu$ is called a chain without zigzags.

RESTRICTION 2. In the case of ordered involuted semigroups satisfying condition (1.2) we may consider axioms $\langle S\rangle$ for those chain systems $S$ only which consist of chains without zigzags.

Motivation. Suppose a chain $\pi$ contains a zigzag $\left(\nu, v^{-1}, \nu\right)$. Replacing the rigzag by $v$ and doing the same with all other zigzags, we obtain a chain $\bar{\pi}$ without zigzags. Olearly, $[\bar{\pi}] \leqslant[\pi]$ is true in every ordered involuted semigroup satisfying (12). It follows that if we replace all chains $\pi$ by $\bar{\pi}$ in $\delta$, we obtain a new chain system $\bar{S}$ such that $\langle\bar{S}\rangle$ implies $\left\langle S^{\prime}\right\rangle$.

It can bo proved that for every net $M_{n}$ there exists only a finite number of different chain systems satisfying restrictions 1 and 2. Thus every net produces a finite number of axioms for the class of representable ordered involuted semigroups. 
It can also be proved that the only new axiom corresponding to $M_{2}$ is $x_{0} \leqslant x_{1} x_{2} \Rightarrow x_{0} \leqslant x_{1} x_{1}^{-1} x_{0}$ (all the other axioms follow from this one and from (5), (6) and (12)). The only new axiom which we obtain considering various nets $M_{3}$ is $x_{0} \leqslant x_{1} x_{2} \& x_{0} x_{2}^{-1} \leqslant x_{3} x_{4} \Rightarrow x_{0} \leqslant x_{1} x_{4}^{-1} x_{4} x_{2}$. The other axioms follow from (12) and the ones just mentioned.

Now we will give a system of quasiidentities for the class of representable involuted semigroups. To this end we suppose that the language of the lower predicate calculus which we use contains the individual variables $x_{0}, x_{1}, \ldots$ and $y_{0}, y_{1}, \ldots$; if $\mu$ is a chain in $M_{n}$, then $[\mu]_{x}$ denotes the polynomial constructed above, while $[\mu]_{y}$ denotes the polynomial constructed in the above fashion where all variables $x_{i}$ are replaced by $y_{i}$. If $S=\left(\mu_{1}, \ldots, \mu_{n-1}, \mu\right)$ is a chain system in a net $M_{n}$, then $\left(S^{\prime}\right)$ is the following quasiidentity:

$$
\begin{aligned}
& {\left[\mu_{1}\right]_{x}=x_{1} x_{2} \& \ldots \&\left[\mu_{n-1}\right]_{x}=x_{2 n-3} x_{2 n-2} \&[\mu]_{x}=y_{0} \&} \\
& \&\left[\mu_{1}\right]_{y}=y_{1} y_{2} \& \ldots \&\left[\mu_{n-1}\right]_{y}=y_{2 n-3} y_{2 n-2} \&[\mu]_{y}=x_{0} \Rightarrow x_{0}=y_{0} .
\end{aligned}
$$

As in the proof of Theorem 5, we can show that conditions $(S)$ for all chain systems $S$ in all nets are equivalent to the conditions of Theorem 2; hence, the following result holds:

THEOREM 6. An involuted semigroup $A$ is representable if and only if $\boldsymbol{A}^{1}$ satisfies the quasiidentities $(S)$ for every chain system $S$ in every net $M_{n}$

As in the case of ordered involuted semigroups, we may consider quasiidentities corresponding to straightened chain systems only.

We have characterized the classes of representable ordered and unordered involuted semigroups by infinite systems of elementary axioms. We conjecture that the classes are not finitely axiomatizable. It would be interesting to find the axioms for our classes of algebraic systems in the form of explicit axiom schemata without any use of "graph-theoretic scaffolding" consisting of nets.

The characterization of representable (ordered) semigrouds which we give below is quite similar to that of (ordered) involuted. semigroups; therefore we will restrict ourselves to outlines of the proofs.

First of all we should modify appropriately the concept of net. A net of order $n(0 \leqslant n)$ is a graph $B_{n}$ with the vertices $\{0,1, \ldots, 2 n+1\}$ and arrows $\{\mathbf{0}, \mathbf{1}, \ldots, \mathbf{3 n}\}$; each arrow leads from "an even vertex to an odd one; $(0,1)$ is an arrow; for every $i, 1 \leqslant i \leqslant n$, there exist uniquely defined vertices $p_{i}, q_{i} \in\{0,1, \ldots, 2 i-1\}$ such that $\left(p_{i}, 2 i+1\right),(2 i, 2 i+1)$, $\left(2 i, q_{i}\right)$ are arrows. It follows that $p_{i}$ is even and $q_{i}$ is odd. The vertices $\{0,1, \ldots, 2 i+1\}$ and the arrows $\{\mathbf{0}, \mathbf{1}, \ldots, 3 i\}$ form a subgraph which is a net. It is called a subnet (of order $i$ ). We denote this fact as follows:
$B_{i}<B_{n}$. Thus there exists a chain of subnets $B_{0}<B_{1}<\ldots<B_{n}$. Clearly, a net is a bipartite graph.

Thus $B_{n}$ is constructed from $B_{n-1}$ by adding two new vertices and three new arrows, as is shown in Fig. 3

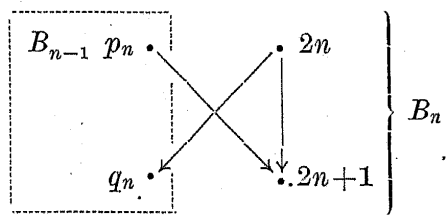

Fig. 3

In $B_{n}$ we will consider only those chains which lead from an even vertex to an odd one. It is easily seen that every such chain consists of an odd number of edges.

If $\boldsymbol{A}=(A ;[], \leqslant)$ is an ordered semigroud, an $\boldsymbol{A}$-metric on a net $B_{n}$ is any mapping $d:\{\mathbf{0}, \mathbf{1}, \ldots, \mathbf{3 n}\} \rightarrow \boldsymbol{A}$. An $\boldsymbol{A}$-metric $d$ is called proper if for every $i, 1 \leqslant i \leqslant n$, there exists a chain $\mu_{i}$ in $B_{i-1}$ leading from $p_{i}$ to $q_{i}$ and such that $d\left(\mu_{i}\right) \leqslant\left[d(3 i-2) d\left((3 i-1)^{-1}\right) d(3 i)\right]$. Eere $3 i-2$ $=\left(p_{i}, 2 i+1\right), 3 \boldsymbol{i}-\mathbf{1}=(2 i, 2 i+1), \mathbf{3} \boldsymbol{i}=\left(2 i, q_{i}\right)$ and $\vec{d}\left(\mu_{i}\right)$ is defined in the obvious fashion: if $\mu_{i}=\left(e_{1}, e_{2}, \ldots, e_{2 k-1}\right)$ then $d\left(\mu_{i}\right)=\left[d\left(e_{1}\right) d\left(e_{2}^{-1}\right) \ldots\right.$ $\left.\ldots d\left(e_{2 k-2}^{-1}\right) d\left(e_{2 k-1}\right)\right]$. Here the edges with odd indices are arrows and edges with even indices are reversed arrows, the product of $2 k-1$ factors is defined inductively: if $k>1$, then $\left.\left[\begin{array}{lll}a_{1} & \ldots & a_{2 k-1}\end{array}\right]=\left[\begin{array}{lll}a_{1} & \ldots & a_{2 k-3}\end{array}\right] a_{2 k-2} a_{2 k-1}\right]$.

A proper $\boldsymbol{A}$-metric $d$ on $B_{n}$ is called regular if $d(0) \leqslant d(\mu)$ for every chain $\mu$ in $B_{n}$ leading from 0 to 1 . Here $0=(0,1)$.

THEOREM 7. An ordered semigroud $\boldsymbol{A}$ is representable if and only if every proper $\boldsymbol{A}$-metric on every net is regular.

Proof. Suppose $\boldsymbol{F}=(F ;[], C)$ is an ordered semigroud of binary relations between the elements of two sets $X$ and $\dot{Y}$. Let $d$ be a proper $F$-metric on a net $B_{n}$ and $\left(x_{0}, x_{1}\right) \in d(0)$ for some $x_{0} \in X$ and $x_{1} \in \bar{Y}$. We are going. to construct a sequence $x_{0}, x_{1}, \ldots, x_{2 n+1}$ such that the terms with even indices belong to $X$, the terms with odd indices belong to $Y$ and $\left(x_{2 i}, x_{2 i+1}\right) \in d((2 i, 2 i+1))$ for all $i, 0 \leqslant i \leqslant n$. We are given $x_{0}$ and $x_{1}$. Suppose that the elements $x_{0}, x_{1}, \ldots, x_{2 i-1}$ satisfying the above condition have already been chosen. Then

$$
\left(p_{i}, q_{i}\right) \in d\left(\mu_{i}\right) \subset\left[d(3 i-2) d\left((3 i-\mathbf{1})^{-1}\right) d(3 i)\right],
$$

i.e. there exist $x_{2 i+1} \in Y$ and $x_{2 i} \in X$ such that $\left(p_{i}, 2 i+1\right) \in d(3 i-2)$, $(2 i, 2 i+1) \in d(\mathbf{3 i}-\mathbf{1}),\left(2 i, q_{i}\right) \in d(\mathbf{3 i})$. Proceeding as above, we choose the terms of the sequence $x_{0}, \ldots, x_{2 n+1}$. 
If $\mu$ is a chain in $B_{n}$ leading from 0 to 1 , then $\left(x_{0}, x_{1}\right) \in d(\mu)$. Since this is true for arbitrary $x_{0} \in X$ and $x_{1} \in Y, d(0) \subset d(\mu)$, i.e. $d$ is regular.

Sufficiency. As in the proof of Theorem 1, we see that it suffices to prove the representability of a finite or countably infinite ordered semigroud $A$ satisfying the condition of Theorem 7 . We construct a finite or infinite sequence $B_{0}<B_{1}<\ldots$ of nets and $d_{0} \subset d_{1} \subset \ldots$ of proper $A$-metrics $\left(d_{i}\right.$ is a metric on $\left.B_{i}\right)$ such that the following condition holds:

(Q) for every chain $\mu$ in $B_{m}$ leading from $2 i$ to $2 j-1$ and for every $a_{1}, a_{2}, a_{3} \in A$ such that $d(\mu) \leqslant\left[a_{1} a_{2} a_{3}\right]$ there exist an $n$ and vertices $2 p$, $2 q-1$ in $B_{n}$ such that $m \leqslant n$. and $d_{n}((2 i, 2 q-1)) \leqslant a_{1}, d_{n}((2 p, 2 q-1))$ $\leqslant a_{2}, d_{n}((2 p, 2 j-1)) \leqslant a_{3}$.

Such sequences may be constructed in a way analogous to that used for constructing sequences satisfying property $(\mathrm{P})$ in the proof of sufficiency of. Theorem 1.

Suppose that such sequences have been constructed and $\partial_{0}(\mathbf{0})=a$. For every $b \in A$ define a binary relation $P_{a}(b)$ between even and odd numbers: $(2 i, 2 j-1) \in P_{a}(b)$ iff $d_{n}(\mu) \leqslant b$ for some $n$, a chain $\mu$ in $B_{n}$ leading from $2 i$ to $2 j-1$. One can easily verify that

$P_{a}\left(\left[a_{1} a_{2} a_{3}\right]\right)=\left[P_{a}\left(a_{1}\right) P_{a}\left(a_{2}\right) P_{a}\left(a_{3}\right)\right]$ and $a_{1} \leqslant a_{2} \Rightarrow P_{a}\left(a_{1}\right) \subset P_{a}\left(a_{2}\right)$.

Suppose that $P(b)$ is a disjoint union (sum) of binary relations $P_{a}(b)$ for all $a \in A$. Then $P\left(\left[a_{1} a_{2} a_{3}\right]\right)=\left[P\left(a_{1}\right) P\left(a_{2}\right) P\left(a_{3}\right)\right]$ and $a_{1} \leqslant a_{2} \Rightarrow P\left(a_{1}\right)$ $\subset P\left(a_{2}\right)$. Now if $P\left(a_{1}\right) \subset P\left(a_{2}\right)$, then $(0,1) \in P_{a_{1}}\left(a_{1}\right) \subset P_{a_{1}}\left(a_{2}\right)$; therefore $d_{n}(\mu) \leqslant a_{2}$ for some chain in $B_{n}$ leading from 0 to 1 . By the regularity of $d_{n}, d_{n}(0) \leqslant d_{n}(\mu) \leqslant a_{2} ;$ therefore, $a_{1}=d_{n}(0) \leqslant a_{2}$, i.e. $a_{1} \leqslant a_{2} \Leftrightarrow P\left(a_{1}\right)$ C $P\left(a_{2}\right)$. It follows that $P$ is an isomorphism of $A$ onto an ordered semigroud of binary relations, i.e. $A$ is representable. Theorem 7 is proved.

A chain system in a net $B_{n}$ is a sequence $\left(\mu_{1}, \ldots, \mu_{n}, \mu\right)$ where $\mu_{i}$ is a chain in $B_{i-1}$ leading from $p_{i}$ to $q_{i}$ and $\mu$ is a chain in $B_{n}$ leading from 0 to 1 . To every chain $v=\left(e_{1}, e_{2}, \ldots, e_{2 k-1}\right)$ there corresponds a polynomial $[\nu]=\left[\left[e_{1}\right]\left[e_{2}\right] \ldots\left[e_{2 k-1}\right]\right]$ where $\left[e_{i}\right]=x_{j}$ if $e_{i}=j$ or $e_{i}=j^{-1}$. If $S$ is a chain system in $B_{n}$, then $\langle S\rangle$ denotes the following formula:

\section{$\left[\mu_{1}\right] \leqslant\left[x_{1} x_{2} x_{3}\right] \&\left[\mu_{2}\right] \leqslant\left[x_{4} x_{5} x_{6}\right] \& \ldots \&\left[\mu_{n}\right] \leqslant\left[x_{3 n-2} x_{3 n-1} x_{3 n}\right] \Rightarrow x_{0} \leqslant[\mu]$}

THEOREM 8. An ordered semigroud is representable if and only if it satisfies the axioms $\langle S\rangle$ for all chain systems. $S$ in all nets $B_{n}$

This theorem can be deduced from Theorem 7 in the same way as Theorem 5 has been deduced from Theorem 1

In what follows $[\mu]_{x}$ denote $[\mu]$ in the above sense and $[\mu]_{y}$ denotes an analogous polynomial in which $x_{i}$ are replaced by $y_{i}$

THEOREM 9. A semigroud is representable if and only if for all chain systems $S=\left(\mu_{1}, \ldots, \mu_{m}, \mu\right)$ and $T=\left(\nu_{1}, \ldots, v_{n}, \nu\right)$ in arbitrary nets $B_{m}$ and $B_{n}$ respectively the following condition $(S, T)$ is satisfied:

$\left[\mu_{1}\right]_{x}=\left[x_{1} x_{2} x_{3}\right] \& \ldots \&\left[\mu_{m}\right]_{x}=\left[x_{3 m-2} x_{3 m-1} x_{3 m}\right] \&[\mu]_{x}=y_{0} \&$

$\&\left[\nu_{1}\right]_{y}=\left[y_{1} y_{2} y_{3}\right] \& \ldots \&\left[v_{n}\right]_{y}=\left[y_{3 n-2} y_{3 n-1} y_{3 n}\right] \&[\nu]_{y}=x_{0} \Rightarrow x_{0}=y_{0}$.

Proof. It can easily be verified that the condition of Theorem 9 means that for all nets $B_{m}$ and $B_{n}$, all proper $A$-metrics $d_{m}$ (for a semigroud $A$ ) and $d_{n}$ on $B_{m}$ and $B_{n}$, respectively, and all chains $\mu$ and $\nu$ in $B_{m}$ and $B_{n}$, each leading from 0 to 1 , the following condition is satisfied: if $d_{m}(\mu)=d_{n}(\mathbf{0})$ and $d_{n}(\nu)=d_{m}(\mathbf{0})$, then $d_{m}(\mathbf{0})=d_{n}(\mathbf{0})$. The proof that a semigroud $A$ is, representable if and only if it satisfies the latter condition is quite analogous to the proof of Theorem 2 and will be omitted for this reason.

Remark 4. Theorem 9 is analogous to Theorem 6 ; however, conditions $(S, T)$ of Theorem 9 are more complicated than those of Theorem 6 , since two nets and two different chain systems $S$ and $T$ are involved. In Theorems 2 and 6 we circumvented this complication by adding an identity to $\boldsymbol{A}$ and considering $\boldsymbol{A}^{1}$-metrics (in particular, the axioms of Theorem 6 should be satisfied by $\left.\boldsymbol{A}^{1}\right)$. We could do the same in Theorem 9 , considering the conditions of the form $(S, S)$; however, in this case they should be satisfied not in $A$ but in an appropriate extension of $\boldsymbol{A}$ (a new element $u$ such that $[u u x]=[x u u]=x$ for all $x \in A \cup\{u\}$ should be added, unless $A$ already has one; this implies that an infinity of new elements of the form $\left[u x_{1} u x_{2} u \ldots u x_{n} u\right]$ should be added).

A chain $\mu$ is called straightened if it does not contain subchains of the form $\left(3 i-2,(3 i-1)^{-1}, 3 i\right)$ or $\left(3 i-2,(3 i-1)^{-1}, 3 i\right)^{-1} ; \mu$ is called without zigzags if it does not contain subchains of the form $\left(\nu, v^{-1}, v\right)$. One can easily verify (using $B_{0}$ only) that a representable ordered semigroud satisfies the condition

$$
x \leqslant[x x x] \text {. }
$$

As in Restrictions 1 and 2 , it can be proved that in Theorems 8 and 9 we may consider straightened chains only; in Theorem 8 we may consider chains without zigzags only, provided the ordered semigroud satisfies (13). The following analogue of Proposition 3 and Corollary 4 holds:

Proposirion 10. Free semigrouds are representable. The class of all representable semigrouds is not a variety of algebras since it is not closed under homomorphisms.

It can be seen that a free semigroud with a set $X$ of free generators is merely the set of all words of odd length over the alphabet $X$ with the following ternary operation:

$$
\left[\left(x_{1} \ldots x_{2 i-1}\right)\left(y_{1} \ldots y_{2 j-1}\right)\left(z_{1} \ldots z_{2 k-1}\right)\right]=x_{1} \ldots x_{2 i-1} y_{2 j-1} \ldots y_{1} z_{1} \ldots z_{2 k-1}
$$


It has been noted in the introduction to this paper that to every involuted semigroup $\boldsymbol{A}=\left(A ; \cdot{ }^{-1}\right)$ there corresponds an associated semigroud $[\boldsymbol{A}]=(A ;[])$ where $\left[a_{1} a_{2} a_{3}\right]=a_{1} a_{2}^{-1} a_{3}$. In the same way, to every ordered involuted semigroup $A$ there corresponds an associated ordered semigroud $[\boldsymbol{A}]$ with the same order relation as in $\boldsymbol{A}$.

Proposition 11. An (ordered) involuted semigroup with identity is representable if and only if the associated (ordered) semigroud is representable.

Proof. The "if" part follows from the obvious fact: isomorphisms of (ordered) involuted semigroups are isomorphisms of the associated (ordered) semigrouds as well.

To prove the "if" part suppose that $A$ is an (ordered) involuted semigroup with the identity 1 and $[A]$ is representable. Let $P$ be an isomorphism of $[A]$ onto an (ordered) semigroud of binary relations between the elements of two sets $X$ and $Y$. Define $Q(a)=P(1)^{-1} \circ P(a)$ for all $a \in A$. Then $Q(a)$ is a binary relation on $X$. Now $Q(a b)=Q(a 1 b)=Q([a 1 b])$ $=P(1)^{-1} \circ P([a 1 b])=P(1)^{-1} \circ P(b) \circ P(1)^{-1} \circ P(a)=Q(b) \circ Q(a) ; Q\left(a^{-1}\right)$ $=Q\left(1 a^{-1} 1\right)=Q([1 a 1])=P(1)^{-1} \circ P([1 a 1])=P(1)^{-1} \circ P(1) \circ P(a)^{-1} \circ P(1)$ $=\left(P(a) \circ P\left(1^{-1}\right) \circ P(1)\right)^{-1} \circ P(1)=P([11 a])^{-1} \circ P(1)=P(a)^{-1} \circ P(1)$ $=\left(P(1)^{-1} \circ P(a)\right)^{-1}=Q(a)^{-1}$. If $Q(a)=Q(b)$, then $P(a)=P([a 11])$ $=P(1) \circ P(1)^{-1} \circ P(a)=P(1) \circ Q(a)=P(1) \circ Q(b)=P(1) \circ P(1)^{-1} \circ P(b)$ $=P([b 11])=P(b)$, hence, $a=b$. If $A$ is ordered, then $a \leqslant b \Rightarrow P(a)$ $\subset P(b) \Rightarrow P(1)^{-1} \circ P(a) \subset P(1)^{-1} \circ P(b) \Rightarrow Q(a) \subset Q(b) \Rightarrow P(1) \circ Q(a) \subset P(1) \circ$ - $Q(b) \Rightarrow P([a 11]) \subset P([b 11]) \Rightarrow P(a) \subset P(b) \Rightarrow a \leqslant b$, i.e. $a \leqslant b \Leftrightarrow Q(a) \subset Q(b)$ It follows that $Q$ is an isomorphism of $\boldsymbol{A}$ onto an (ordered) semigroud of binary relations. Proposition' 11 is proved.

It would be interesting to know whether Proposition 11 is valid for arbitrary (ordered) involuted semigroups.

\section{References}

[1] П. А. Бредихин, Представление упорлдоченных ильолютированных полугрупи, Известия высшг. учебн. завед., Матем. 1974.

[2] В. В. Вагнер, Теория обобщенных груд и обобценных групn, Матем. сборник 32 (1953) crp. 545-632.

[3] R. Croisot, Algèbre de relations et hypergroupes partiels, C. R. Acad. Sci. Paris 228 (1949), pp. 1181-1182.

[4] Л. М: Глускин, Представления полугруд, В сборнике статей „Памятхи Н. Г. Чеботарёва" “, Казань, 1964, стр. 44-59.

[5] К. А. Зарецкий, Абстрактная характеристика класса полугрупп иастиинь реблексивнызх бинарных отношений, Сибирский матем. журнал 8 (1967), стр. 1299-1306.

[6] - Абстрактная характеристика полугруппы всех бинарных опнноиений, Учёные записки Ленинградск. гос. педагог. института им. А. И. Герцена 183 (1958), стр. 251-263.

[7] B. Jónsson, Representation of modular lattices and of relation algebras, Trans. Amer. Math. Soc. 92 (1959), pp. 449-464.
[8] R. M'C Kenzie, A letter to the author (June 22, 1966).

[9] J. C. C. McKinsey, Postulates for the calculus of binary relations, J. Symbolic Logic 5 (1940), pp. 85-97.

[10] B. M. Schein, Relation algebras, Bull. Acad. Polon. Sci. Sér. Sci. Math. Astronom. Phys. 13 (1965), pp. 1-5.

[11] - Relation algebras and function semigroups, Semigroup Forum I (1970), pp. 1-62.

[12] Б. М. Шайн, Инволтотированные полугруппы полных бинарных отноиений, Поклады Акад. Наук СССР 156 (1964), стр. 1300-1303.

[13] - О некоторых классах полугрупп бинарных отношений, Сибирский матем. журнал 6 (1965), стр. 616-635.

[14] - Aтомные полугруды и инволютированные полугруппы, Известия выст. учебн. завед., Матем. 3 (1965), стр. 172-184.

[15] - Симмепрические обобиенные груды, Научные доклады высшей школы, Физ.-матем. науки 2 (1), (1959), стр. 88-93.

[16] - Полугруппы прямоугольных бинарных опноиений, Доклады Акад. Наук СССР 165 (1965), crp. 1011-1014.

[17] A. Tarski, On the calculus of relations, J. Symbolic Logic 6.(1941), pp. 73-89.

[18] - Contributions to the theory of models II, Proc. Koninkl. Nederl. Akad. Wetensch. A57 (1954), pp. 582-588.

[19] Свердловская тетрадь, Нерециенные задачи теории полугрупn, Свердловск, 1969 the English translation: The Sverdlorsk Tetrad, Semigroup Forum 4 (1972), pp. 274-280. 